\title{
A Model Predictive Control Approach to Microgrid Operation Optimization
}

\author{
Alessandra Parisio, Member, IEEE, Evangelos Rikos, and Luigi Glielmo, Senior Member, IEEE
}

\begin{abstract}
Microgrids are subsystems of the distribution grid, which comprises generation capacities, storage devices, and controllable loads, operating as a single controllable system either connected or isolated from the utility grid. In this paper, we present a study on applying a model predictive control approach to the problem of efficiently optimizing microgrid operations while satisfying a time-varying request and operation constraints. The overall problem is formulated using mixed-integer linear programming (MILP), which can be solved in an efficient way by using commercial solvers without resorting to complex heuristics or decompositions techniques. Then, the MILP formulation leads to significant improvements in solution quality and computational burden. A case study of a microgrid is employed to assess the performance of the online optimization-based control strategy and the simulation results are discussed. The method is applied to an experimental microgrid located in Athens, Greece. The experimental results show the feasibility and the effectiveness of the proposed approach.
\end{abstract}

Index Terms-Microgrids, mixed logical dynamical systems, mixed-integer linear programming (MILP), model predictive control (MPC), optimization.

\section{INTRODUCTION}

$\mathbf{T}$ HE NEED to satisfy in sustainable ways, the increasing energy demand requires active energy distribution networks, i.e., distribution networks with the possibility of bidirectional power flows controlling a combination of distributed energy resources (DERs), such as distributed generators (DGs) and renewable energy devices. In this scenario, the microgrid concept is a promising approach. It is an integrated energy system consisting of interconnected loads and DERs, which can operate in parallel with the grid or in an intentional island mode, see [1] and [2]. A typical microgrid comprises: 1) storage units; 2) DGs, which are controllable units;

Manuscript received May 16, 2013; revised September 25, 2013; accepted November 20, 2013. Manuscript received in final form December 17, 2013. Date of publication January 9, 2014; date of current version July 24, 2014 This work was supported in part by the Distributed Energy Resources Research Infrastructures Project, in part by the Sustainable-Smart Grid Open System for the Aggregated Control, Monitoring and Management of Energy Project, in part by the European Institute of Technology Information and Communication Technology Labs, and in part by the Swedish Governmental Agency for Innovation Systems. Recommended by Associate Editor A. Horch.

A. Parisio is with the ACCESS Linnaeus Center and the Automatic Control Laboratory, School of Electrical Engineering, KTH Royal Institute of Technology, Stockholm SE-100 44, Sweden (e-mail: parisio@kth.se).

E. Rikos is with the Department of PVs and DER systems, Center for Renewable Energy Sources and Saving, Athens 10680, Greece (e-mail: vrikos@gres.gr).

L. Glielmo is with the Department of Engineering, Universitàdegli Studi del Sannio, Benevento 82100, Italy (e-mail: glielmo@unisannio.it).

Color versions of one or more of the figures in this paper are available online at http://ieeexplore.ieee.org.

Digital Object Identifier 10.1109/TCST.2013.2295737
3) renewable energy resources (RESs), which are noncontrollable devices; and 4) controllable loads, which can be curtailed (or shed) when it is more convenient. In addition, a microgrid can purchase and sell power to and from its energy suppliers.

One of the main motivations behind using microgrids is that they are capable of managing and coordinating DGs, storages, and loads in a more decentralized way reducing the need for the centralized coordination and management [3]. Hence, the optimization of the microgrid operations is extremely important in order to cost efficiently manage its energy resources [2], [4].

In this scenario, new modeling requirements are needed, e.g., storage modeling must be incorporated into the operation planning problem in order to coordinate storage use with RESs generation and energy prices, and address the complexity of the charging/discharging schedule [5]. It is important to notice that there are no current modeling tools including controllable loads and energy storage modeling in a smart grid environment [6].

Notice further that a complete formulation of microgrid optimal operation planning problem includes modeling of storages, demand side policies for controllable loads [demand side management (DSM)], power exchange with the utility grid. Microgrid modeling needs both continuous (such as storage charge/discharge rates) and discrete (such as ON/OFF states of DGs and DSM controlled loads) decision variables, and the problem is generally formulated as a mixed integer nonlinear problem (MINLP) (see [7]-[9]), for which there is no exact solution technique.

Another relevant aspect in microgrid management, which further complicates it, is coping with uncertainty in the energy demand, RESs generation and energy prices.

The modeling capabilities and the computational advances of mixed integer problem (MIP) algorithms, have led several independent system operators and regional transmission organizations to implement MIP-based solution methods in order to find a better solution to solve day-ahead and real-time market problems [10]; namely, not solving unit commitment problems to complete optimality can cause several issues [11].

Therefore, it is necessary to find a tractable formulation of the microgrid operation optimization problem, which includes the specific key features of a microgrid.

In this paper, we tackle the optimal operation planning of a microgrid. This problem aims at minimizing the overall microgrid operating costs to meet predicted load demand of a certain period (typically one day) while satisfying complex operational constraints, such as the energy balance, and 
controllable generators minimum operation time and minimum stop time.

\section{A. Literature Review}

Due to the microgrid optimization problem complexity and because of the large economic benefits that could result from its improved solution, considerable attention is being devoted to the development of better optimization algorithms and suitable modeling frameworks. Metaheuristics and heuristics have been proposed to solve the power dispatch problem for microgrids, such as genetic algorithms [12], evolutionary strategies, and tabu search algorithms [13].

Studies have suggested that microgrids can achieve high performance through: 1) advanced control algorithms accounting for system uncertainty and based on predicted future conditions; 2) deployment of demand response; 3) optimal use of storage devices in order to compensate the physical imbalances; and 4) applying optimal instead of heuristicbased approaches (see [14]-[17] and the references therein). Typically the proposed approaches either are computationally intensive and not suitable for real-time applications, or can produce suboptimal solutions, see [17]-[19]. Further, in the aforementioned works, either the optimization problem stays nonlinear or other important features, such as minimum up and down times and demand side programs, are neglected. For instance, in [20] the microgrid optimization problem is tackled by solving several MINLPs (e.g., one separate problem for unit commitment, one for storage management, and so on).

Recently, model predictive control (MPC) has drawn the attention of the power system community due to several factors [21]: 1) it is based on future behavior of the system and predictions, which is attractive for systems greatly dependent on demand and renewable energy generation forecasts; 2) it provides a feedback mechanism, which makes the system more robust against uncertainty; and 3) it can handle power system constraints, such as generator capacity and ramp rate constraints. An MPC method has been commonly proposed to solve the unit commitment problem with wind power generation (see [22]). Further, an MPC-based dynamic voltage and var control scheme has just been developed for reactive power control, in order to avoid unstable voltage conditions in microgrids, especially during islanded mode operation with no support from the utility grid [23].

Some works can be found in the literature that address MPC for optimal dispatch in power systems. The authors in [24] model a combined cycle power plant by utilizing hybrid systems in order to describe both the continuous/discrete dynamics and the switching between different operating conditions. Then the plant operations are economically optimized through MPC by taking into account the time variability of both prices and electricity/steam demands. Ferrari-Trecate et al. [24] propose an MPC algorithm to solve the economic dispatch problem with large presence of intermittent resources. However, many microgrid key features, such as demand side programs, storages and ON/OFF generators status are not considered. In [26] and [27], an MPC is applied to managing the energy flows inside a household system equipped with a microcombined heat and power unit. In addition, the household can buy and sell electricity from/to the energy supplier and heat and electricity can be stored in specific storage devices. Hooshmand et al. [28] and Xia et al. [29] apply an MPC framework to solve the dynamic economic dispatch, which aims at minimizing the generation cost over a particular time interval (the dispatch interval). Then, the goal is to decide the power dispatch in order to meet the demand at minimum cost subject to limits on power generation and ramp rates.

In [30], a supervisory control system via MPC is designed for a wind/solar energy generation system, which computes the power references for the wind and solar subsystems at each sampling time while minimizing a suitable cost function. The power references are sent to two local controllers, which drive the two subsystems to the requested power references. In [31], the centralized supervisory MPC controller is replaced with two distributed supervisory MPC controllers, each responsible for providing optimal reference trajectories to the local controller of the corresponding subsystem. The supervisory optimization problem solved is nonlinear and nonconvex, and several issues are not addressed, e.g., system startup or shut down.

In [32], an energy management system based on a rolling horizon strategy is proposed for an islanded microgrid comprising photovoltaic (PV) panels, two wind turbines, a diesel generator, and an energy storage system. The problem includes several nonlinear constraints associated with the modeling the two controllable units (the diesel generator and the storage system). The nonlinear constraints are approximated by piecewise linear models and the optimization problem is solved using mixed-integer linear programming (MILP), yielding suboptimal solutions.

Eventually, we would like to remark that when storage elements are considered, generally the storage is modeled as a discrete-time first-order system with two continuous variables representing charging and discharging power multiplied by suitable, and different, charging and discharging efficiencies. That approach does not rule out the possibility that the optimal solution contemplates simultaneous charging and discharging of the storage, a physically unrealizable policy. Such outcome may occur as the mathematical consequence of unpredicted RES generated power surplus, bounds on the exchanged power with the utility grid, and costs of the storage level. This issue has been never discussed in the corresponding studies. Because of the different multiplicative factor in charging and discharging, one continuous variable, which can take both positive and negative values, cannot represent correctly the storage behavior. Similarly the interaction with the utility grid should be modeled so as to prevent simultaneous selling and purchasing under certain market circumstances.

\section{B. Main Assumptions}

In a microgrid control structure, several aspects should be addressed, whose requirements involve different control approaches and different time scales: 1) fast electrical control of the phase, frequency, and voltage of individual components 
on time scales of seconds or less and 2) unit commitment and economic dispatch of all generating units and storages, RES generation and load forecasting, demand-side optimization, and energy exchanges with the utility grid on longer time scales (e.g., hours). Thus, a reasonable approach is to develop a hierarchical control structure; a centralized, high level controller is on the top of the hierarchy, while the second level includes load controllers and DERs controllers [33]. The aim of the high level control algorithm is to generate suitable set points for all sources and storages so that economically optimized power dispatch will be performed and a given demand is met. The local controllers have to guarantee that the system tracks the power reference values.

We point out the following.

1) In a hierarchy of controllers, we aim at a high level optimization of microgrid operations; voltage stability, power quality, and frequency are supposed to have higher priority and to be controlled at the lower control level. Remarkably, we focus our study on the gridconnected mode, which implies that the frequency of the microgrid is maintained within a tight range by the utility grid. Further, by limiting the line capacity in the optimization problem, the steady-state power quality is preserved, i.e., our approach does not violate the voltage limits according to the grid and does not cause line congestion.

2) The high level controller deals with the long-term behavior of the system and is very weakly dependent on the transient behavior of the fast dynamics. Then, a steadystate assumption for microgrid components can be safely made without much loss of accuracy.

3) The microgrid high level controller has knowledge of the managed network; it knows the existing generation capacity, storage capacity, network constraints, market energy prices, and bilateral contracts.

4) The microgrid operator is the unique entity in charge of management, aimed at optimizing profits. It can take economic decisions, such as to sell or buy energy depending on local generation capabilities and costs and the energy prices.

5) Due to constant sampling time $\Delta T=t_{k+1}-t_{k}$, there exists a constant ratio between energy and power at each interval.

\section{Main Contributions}

We present a control-oriented approach to microgrid modeling and high level optimization and propose the use of MPC in combination with MILP [34], [35]. The microgrid operations are decided on the basis of predictions of future behavior of the system and renewable power generation and demand forecasts.

To guarantee a feasible behavior for storage and grid interaction (e.g., nonsimultaneous charging and discharging, buying, and selling), we utilize the approach described in [36] and use the mixed logical dynamical framework. We would like to remark that in the proposed problem formulation, only generators' fuel consumption and emission functions are approximated in case they must be expressed as nonlinear functions, which is not always needed for microgrid components. We assume nonlinear generators' fuel consumption and emission functions in order to state the problem formulation as general as possible.

In our approach, we strove to include as many details as possible, and to use and maintain the microgrid optimization problem solvable without resorting to any decomposition techniques or heuristics. Further, we modeled the generators technical and physical features by using a number of constraints and variables as low as possible.

Further, a feedback mechanism is introduced (MPC), which compensates for the uncertainty in microgrid operations associated with: 1) the RES power outputs; 2) the time-varying load; and 3) time-varying energy prices. In addition, since the optimum is reached in a reasonable computation time, a shorter sampling time can be used (e.g. $15 \mathrm{~min}$, rather than 30 or 60), which allows to compute more accurate and effective solutions.

This paper extends the preliminary study presented in [37] by: 1) discussing experimental results obtained from a microgrid located in Athens, Greece; 2) including further simulation results and a comparison with a heuristic algorithm; and 3) taking the costs of the power exchanged with the storage unit into account in the objective function. In addition, renewable power generation and demand forecasts are computed.

In summary, our contributions are: 1) the development of a novel model of the overall microgrid system adopting a formalized modeling approach, which is suitable to be used in online optimization schemes; 2) the development of an MPC scheme for minimizing the microgrid running costs; 3) the presentation of simulation results showing the effectiveness of the proposed optimization routine; and 4) application of the method to an experimental microgrid located in Athens, Greece, and the estimation of all the parameters and costs required to carry out the experiments.

\section{Outline}

This paper is further organized as follows: 1) the microgrid system is described and the microgrid modeling approach is outlined in Section II; 2) the operations optimization is then described in Section III; 3) some simulation results are discussed in Sections IV and V and in Section VI the experimental results are presented; and 4) finally, conclusions are drawn in Section VII.

\section{E. Nomenclature}

The forecasts, the parameters and the decision variables used in the proposed formulation are described, respectively, in Tables I-III, where, for simplicity, we omit the subscript $i$ when referring to the $i$ th unit.

We remark that the fuel consumption cost for a DG unit is traditionally assumed to be a quadratic function of the form $C^{\mathrm{DG}}(P)=a_{1} P^{2}+a_{2} P+a_{3}$.

In the following sections, vectors and matrices are denoted in bold. 
TABLE I

PARAMETERS

\begin{tabular}{|c|c|}
\hline Parameters & Description \\
\hline$N_{g}, N_{l}, N_{c}$ & $\begin{array}{l}\text { number respectively of DG units, critical loads } \\
\text { and controllable loads }\end{array}$ \\
\hline$C^{D G}(P)$ & $\begin{array}{l}\text { fuel consumption cost curve of a DG unit } \\
\text { depending on generated power }\end{array}$ \\
\hline $\begin{array}{l}a_{1}, a_{2}, a_{3} \\
O M\end{array}$ & $\begin{array}{l}\text { cost coefficients of } C^{D G}(P)\left[€ /(\mathrm{kWh})^{2}, € / \mathrm{kWh}, €\right] \\
\text { operating and maintenance cost of a DG unit }[€ / \mathrm{h}]\end{array}$ \\
\hline$O M^{b}$ & $\begin{array}{l}\text { operating and maintenance cost of the power } \\
\text { exchanged with the storage unit }[€ / \mathrm{kWh}]\end{array}$ \\
\hline$R_{\max }$ & ramp up limit of a DG unit $[\mathrm{kW} / \mathrm{h}]$ \\
\hline$T^{\text {up }}, T^{\text {down }}$ & minimum up and down time of a DG unit [time-units] \\
\hline$x^{s b}$ & storage 'physiological' energy loss $[\mathrm{kWh}]$ \\
\hline$x_{\min }^{b}, x_{\max }^{b}$ & $\begin{array}{l}\text { minimum, maximum energy level } \\
\text { of the storage unit }[\mathrm{kWh}]\end{array}$ \\
\hline$C^{b}$ & storage output power limit $[\mathrm{kW}]$ \\
\hline$T^{g}$ & $\begin{array}{l}\text { maximum interconnection power flow limit } \\
\text { (at the point of common coupling) }[\mathrm{kW}]\end{array}$ \\
\hline$P_{\min }, P_{\max }$ & minimum, maximum power level of a DG unit $[\mathrm{kW}]$ \\
\hline $\begin{array}{l}\eta^{c}, \eta^{d} \\
\beta_{\min }, \beta_{\max }\end{array}$ & $\begin{array}{l}\text { storage charging and discharging efficiencies } \\
\text { minimum, maximum allowed curtailment } \\
\text { of a controllable load }\end{array}$ \\
\hline$c^{S U}, c^{S D}$ & start-up, shut-down costs of a DG unit $[€]$ \\
\hline$D^{c}$ & preferred power level of a controllable load $[\mathrm{kW}]$ \\
\hline$\rho_{c}$ & penalty weight on curtailments \\
\hline
\end{tabular}

TABLE II

FORECASTS

\begin{tabular}{|l|l|}
\hline Forecasts & Description \\
\hline$P^{\text {res }}$ & sum of power production from RES $[\mathrm{kW}]$ \\
$D$ & power level required from a critical load $[\mathrm{kW}]$ \\
$c^{P}, c^{S}$ & purchasing, selling energy prices [€/kWh] \\
\hline
\end{tabular}

TABLE III

DECISION AND LOGICAL VARIABLES

\begin{tabular}{|l|l|}
\hline Variables & Description \\
\hline$\delta$ & off(0)/on(1) state of a DG unit \\
$\delta^{b}$ & discharging(0)/charging(1) mode of the storage unit \\
$\delta^{g}$ & exporting(0)/importing(1) mode to/from the utility grid \\
$P$ & power level of a DG unit [kW] \\
$P^{b}$ & power exchanged (positive for charging) \\
& with the storage unit [kW] \\
$P^{g}$ & importing(positive)/exporting(negative) power level \\
& from/to the utility grid [kW] \\
$x^{b}$ & stored energy level [kWh] \\
$\beta$ & curtailed power percentage \\
\hline
\end{tabular}

\section{System Description, Modeling, AND CONSTRAINTS}

Here, we briefly describe the key features of the microgrid architecture considered in this paper and associate a possible modeling setup with the goal of maintaining the problem tractable and suitable for real-time computation.

\section{A. Loads}

We consider two types of loads:

1) critical loads, i.e., demand levels related to essential processes that must be always met;

2) controllable loads, i.e., loads that can be reduced or shed during supply constraints or emergency situations (e.g., standby devices, day-time lighting).
In demand response programs, the customers specify level of curtailment of the controllable loads. The controllable loads have a preferred level, but their magnitude is flexible so that the demand level can be lowered when it is convenient or necessary (e.g., in islanded mode). This leads to users' discomfort, hence a certain cost is associated with the load curtailment/shedding (a penalty for the microgrid). We define a continuous-valued variable, $0 \leq \beta_{c}(k) \leq 1$, associated to each controllable load $c$ and to each sampling time $k$. This variable represents the percentage of preferred power level to be curtailed at time $k$ in order to keep the microgrid operations feasible (e.g., in islanded mode) or more economically convenient. If no curtailment is allowed at a certain time $\hat{k}$, an equality constraint can be set, $\beta_{c}(\hat{k})=0$.

\section{B. Storage Dynamics}

For a storage unit, denoting by $x^{b}(k)$ the level of the energy stored at time $k$ (divided by $\Delta T$ ) and by $P^{b}(k)$ the power exchanged with the storing device at time $k$, we consider the following discrete time model of a storage unit:

$$
x^{b}(k+1)=x^{b}(k)+\eta P^{b}(k)-x^{s b}
$$

where

$$
\eta= \begin{cases}\eta^{c}, & \text { if } P^{b}(k)>0 \text { (charging mode) } \\ 1 / \eta^{d}, & \text { otherwise (discharging mode) }\end{cases}
$$

with $0<\eta^{c}, \eta^{d}<1$.

The charging and discharging efficiencies account for the losses and $x_{\mathrm{sb}}$ denotes a constant stored energy degradation in the sampling interval. If the power exchanged at time $k$, $P^{b}(k)$, is greater than zero, this will be charging the storage device, otherwise the storage device will be discharged.

Using the standard approach described in [36], we introduce a binary variable $\delta^{b}(k)$ and an auxiliary variable $z^{b}(k)=\delta^{b}(k) P^{b}(k)$ to model the following logical condition and the storage dynamics:

$$
P^{b}(k) \geq 0 \Longleftrightarrow \delta^{b}(k)=1
$$

and

$$
x^{b}(k+1)= \begin{cases}x^{b}(k)+\eta^{c} P^{b}(k)-x^{s b}, & \text { if } \delta^{b}(k)=1 \\ x^{b}(k)+1 / \eta^{d} P^{b}(k)-x^{s b}, & \text { otherwise }\end{cases}
$$

then, we express the logical conditions as mixed integer linear inequalities. By collecting such inequalities, we can rewrite the storage dynamics and the corresponding constraints in the following compact form (the interested reader is referred to [36] for guiding details):

$$
\begin{aligned}
& x^{b}(k+1)=x^{b}(k)+\left(\eta^{c}-1 / \eta^{d}\right) z^{b}(k)+1 / \eta^{d} P^{b}(k)-x^{s b} \\
& \text { subject to } \mathbf{E}_{\mathbf{1}}{ }^{b} \delta^{b}(k)+\mathbf{E}_{\mathbf{2}}{ }^{b} z^{b}(k) \leq \mathbf{E}_{\mathbf{3}}{ }^{b} P^{b}(k)+\mathbf{E}_{\mathbf{4}}{ }^{b}
\end{aligned}
$$

where the column vectors $\mathbf{E}_{\mathbf{1}}^{b}, \mathbf{E}_{\mathbf{2}}{ }^{b}, \mathbf{E}_{\mathbf{3}}{ }^{b}, \mathbf{E}_{\mathbf{4}}{ }^{b}$ are easily derived from the six mixed integer linear inequalities modeling the if...then conditions described in (3) and the auxiliary variable

$$
z^{b}(k)=\delta^{b}(k) P^{b}(k)
$$

which hides a nonlinearity. The mixed integer inequalities are provided in the Appendix C, (18) and (19) with $m=-C^{b}$, 
$M=C^{b}, f(k)=P^{b}(k)$, and $\delta=\delta^{b}(k)$. For example, the logical condition (3) can be rewritten as [see (18) in Appendix C]

$$
\begin{cases}C^{b} \delta^{b}(k) & \leq P^{b}(k)+C^{b} \\ -\left(C^{b}+\varepsilon\right) \delta^{b}(k) & \leq-P^{b}(k)-\varepsilon .\end{cases}
$$

Then, the first two elements of the column vectors in (4) are

$$
\begin{aligned}
& \mathbf{E}_{\mathbf{1}}^{\mathbf{b}^{\prime}}=\left[\begin{array}{lll}
C^{b}-\left(C^{b}+\varepsilon\right) & \cdots
\end{array}\right] \\
& \mathbf{E}_{\mathbf{2}}^{\mathbf{b}^{\prime}}=\left[\begin{array}{lll}
0 & 0 & \cdots
\end{array}\right] \\
& \mathbf{E}_{\mathbf{3}}^{\mathbf{b}^{\prime}}=\left[\begin{array}{lll}
1 & -1 & \cdots
\end{array}\right] \\
& \mathbf{E}_{\mathbf{4}}^{\mathbf{b}^{\prime}}=\left[\begin{array}{lll}
C^{b} & -\varepsilon & \cdots
\end{array}\right] .
\end{aligned}
$$

The other elements are obtained by imposing the inequalities (19) of Appendix C to the variable $z^{b}(k)$, with $f(k)=P^{b}(k)$ and $\delta=\delta^{b}(k)$. Further details are provided in the Appendixes.

The balance between energy production and consumption must be met at each time $k$, so the following equality constraint is imposed:

$$
\begin{aligned}
P^{b}(k)= & \sum_{i=1}^{N_{g}} P_{i}(k)+P^{\mathrm{res}}(k)+P^{g}(k) \\
& -\sum_{j=1}^{N_{l}} D_{j}(k)-\sum_{h=1}^{N_{c}}\left[1-\beta_{h}(k)\right] D_{h}^{c}(k) .
\end{aligned}
$$

If we collect all decision variables in the vector $\mathbf{u}(k)$ and all known disturbances (obtained by forecasts) in the vector $\mathbf{w}(k)$, we can rewrite $P^{b}(k)$ as follows:

$$
P^{b}(k)=\mathbf{F}^{\prime}(k) \mathbf{u}(k)+\mathbf{f}^{\prime} \mathbf{w}(k)
$$

with

$$
\begin{aligned}
\mathbf{u}(k) & =\left[\begin{array}{llll}
\mathbf{P}^{\prime}(k) & P^{g}(k) & \boldsymbol{\beta}^{\prime}(k) & \boldsymbol{\delta}^{\prime}(k)
\end{array}\right]^{\prime} \in \mathbb{R}^{N_{u}} \times\{0,1\}^{N_{g}} \\
\mathbf{w}(k) & =\left[\begin{array}{lll}
P^{\mathrm{res}}(k) & \mathbf{D}^{\prime}(k) & \mathbf{D}^{\mathbf{c}^{\prime}}(k)
\end{array}\right]^{\prime} \in \mathbb{R}^{N_{w}}
\end{aligned}
$$

where $N_{u}=N_{g}+1+N_{c}, N_{w}=1+N_{l}+N_{c} ; \mathbf{P}(k)$, $\boldsymbol{\delta}(k), \mathbf{D}(k), \mathbf{D}^{\mathbf{c}}(k)$, and $\boldsymbol{\beta}(k)$ are column vectors containing, respectively, all the power levels, the generators OFF-/ONstates, the critical demand, the power levels of the controllable loads, and the curtailments. The vectors $\mathbf{F}^{\prime}(k)$ and $\mathbf{f}^{\prime}$ are provided in Appendix A.

Thus, the storage level can be expressed as an affine function by substituting (8) in (4) as follows:

$$
\begin{aligned}
x^{b}(k+1)= & x^{b}(k)+\left(\eta^{c}-1 / \eta^{d}\right) z^{b}(k) \\
& +1 / \eta^{d}\left[\mathbf{F}^{\prime}(k) \mathbf{u}(k)+\mathbf{f}^{\prime} \mathbf{w}(k)\right]-x^{s b} .
\end{aligned}
$$

\section{Interaction With the Utility Grid}

When grid connected, the microgrid can sell and purchase energy from/to the utility grid. By following the same procedure outlined above, we introduce a binary variable $\delta^{g}(k)$ and an auxiliary variable $C^{g}(k)$ to model the possibility either to purchase from or to sell energy to the utility grid. The following logical statements hold:

$$
P^{g}(k) \geq 0 \Longleftrightarrow \delta^{g}(k)=1
$$

and

$$
C^{g}(k)= \begin{cases}c^{P}(k) P^{g}(k), & \text { if } \delta^{g}(k)=1 \\ c^{S}(k) P^{g}(k), & \text { otherwise }\end{cases}
$$

again, we express the if ... then conditions as mixed integer linear inequalities. Then, the purchasing/selling microgrid behavior can be expressed by the following mixed integer linear inequalities in a compact form:

$$
\mathbf{E}_{\mathbf{1}}{ }^{g} \delta^{g}(k)+\mathbf{E}_{\mathbf{2}}{ }^{g} C^{g}(k) \leq \mathbf{E}_{\mathbf{3}}{ }^{g}(k) P^{g}(k)+\mathbf{E}_{\mathbf{4}}{ }^{g} .
$$

The column vectors $\mathbf{E}_{\mathbf{1}}{ }^{g}, \mathbf{E}_{\mathbf{2}}{ }^{g}, \mathbf{E}_{\mathbf{3}}{ }^{g}(k), \mathbf{E}_{\mathbf{4}}{ }^{g}$ are provided in Appendix A. The matrix $\mathbf{E}_{\mathbf{3}}{ }^{g}(k)$ is generally time-varying due to the time varying energy prices. We recall that the interaction with the utility grid is allowed only when the microgrid is in grid-connected mode.

\section{Generator Operating Conditions}

The operating constraints, at each sampling time $k$, on the minimum amount of time for which a controllable generation unit must be kept ON/OFF (minimum up/down times) can be expressed by the following mixed integer linear inequalities without resorting to any additional variable:

$$
\begin{aligned}
& \delta_{i}(k)-\delta_{i}(k-1) \leq \delta_{i}(\tau), \quad(\text { OFF/ON switch }) \\
& \delta_{i}(k-1)-\delta_{i}(k) \leq 1-\delta_{i}(\tau), \quad(\text { ON/OFF switch })
\end{aligned}
$$

with $i=1, \ldots, N_{g}, \tau=k+1, \ldots, \min \left(k+T_{i}^{\text {up }}-1, T\right)$ whether we consider the constraints on the minimum up time or $\tau=k+1, \ldots, \min \left(k+T_{i}^{\text {down }}-1, T\right)$ otherwise.

Consider, for instance, the $i$ th unit at time step $\hat{k}$, with $\delta_{i}(\hat{k}-1)=0$, meaning that the unit was ofF during the previous sampling period. If the value 1 is assigned to the optimization variable $\delta_{i}(\hat{k})$, the first $T_{i}^{\mathrm{up}}-1$ constraints in (11) will force all the binary optimization variables corresponding to the unit ON-/OFF-state to be equal to 1 for the next $T_{i}^{\mathrm{up}}-1$ sampling times. Namely, for $T_{i}^{\mathrm{up}}=3$

$$
\begin{aligned}
& \delta_{i}(\hat{k})-\delta_{i}(\hat{k}-1) \leq \delta_{i}(\hat{k}+1) \\
& \delta_{i}(\hat{k})-\delta_{i}(\hat{k}-1) \leq \delta_{i}(\hat{k}+2)
\end{aligned}
$$

which forces the right-hand side of the inequalities (12) to be equal to 1 in order to satisfy the constraints.

We also model the DG unit startup and shutdown behavior in order to account for the corresponding costs. For this reason, two auxiliary variables, $S U_{i}(k)$ and $S D_{i}(k)$ are introduced, representing, respectively, the startup and the shutdown cost for the $i$ th DG generation unit at time $k$. These auxiliary variables must satisfy the following mixed integer linear constraints:

$$
\begin{aligned}
& S U_{i}(k) \geq c_{i}^{S U}(k)\left[\delta_{i}(k)-\delta_{i}(k-1)\right] \\
& S D_{i}(k) \geq c_{i}^{S D}(k)\left[\delta_{i}(k-1)-\delta_{i}(k)\right] \\
& S U_{i}(k) \geq 0 \\
& S D_{i}(k) \geq 0
\end{aligned}
$$

with $i=1, \ldots, N_{g}$ (see [38] and the references therein). 


\section{OptimizATiON PROBlem}

The microgrid optimal operational planning consists in taking decisions on how to optimally schedule internal production by generators, storage, as well as controllable loads, to cover the microgrid demand and minimize the generators' running costs and the cost of imported electricity from the utility grid in the next hours or day. At every time step, the microgrid controller must take high level decisions about:

1) when should each generation unit be started and stopped (unit commitment);

2) how much should each unit generate to meet this load at minimum cost (economic dispatch);

3) when should the storage device be charged or discharged;

4) when and how much energy should be purchased from or sold to the utility grid (when the microgrid is in the grid-connected mode);

5) curtailment schedule (which controllable loads must be shed/curtailed and when);

6) how much energy has to be stored.

Using the modeling approach of Section II, the problem can be formulated as a MILP optimization problem, which generates an optimal plan. This plan will be subject to uncertainty, the model will be imperfect, the system state will not evolve as predicted. The single MILP is an open loop solution, which does not account for these uncertainties. A possible remedy is to embed MILP optimizations within an MPC framework, so that a feedback control law can be implemented and the uncertainty can be potentially compensated. In absence of uncertainty, these two solutions coincide.

In order to formulate the MPC problem, we next define the cost function associated with the MILP.

\section{A. Linear Approximation of Fuel Consumption Cost Function}

Since experience has shown that mixed integer linear programs are computationally more efficient than quadratic programs [39], the fuel cost function of a DG generator, $C^{\mathrm{DG}}(P)=a_{1} P^{2}+a_{2} P+a_{3}$ is approximated by the max of affine functions without introducing binary variables [40]

$$
C^{\mathrm{DG}}(P) \approx \max _{j=1, \ldots, n}\left\{S_{j} P+s_{j}\right\}=\|\mathbf{S} P+\mathbf{s}\|_{\infty}
$$

where $P$ is the generated power, and $S$ and $s$ are obtained by linearizing the function at $n$ points (the subscript $j$ extracts the $j$ th row of $S$ and $s$ ).

\section{B. Cost Function}

Microgrid economic optimization is achieved by choosing an objective function representing the operating costs to be minimized. The following quadratic function includes costs associated with energy production and startup and shutdown decisions, along with possible earnings and curtailment penalties:

$$
\begin{aligned}
& \sum_{k=0}^{T-1} \sum_{i=1}^{N_{g}}\left[C_{i}^{\mathrm{DG}}\left(P_{i}(k)\right)+O M_{i} \delta_{i}(k)+S U_{i}(k)+S D_{i}(k)\right] \\
& \quad+\mathrm{OM}^{b}\left[2 z^{b}(k)-P^{b}(k)\right]+C^{\mathrm{g}}(k)+\rho_{c} \sum_{h=1}^{N_{c}} \beta_{h}(k) D_{h}^{c}(k)
\end{aligned}
$$

where $k$ is the time instant, $T$ is the length of the prediction horizon, and $2 z^{b}(k)-P^{b}(k)$ models the absolute value of the power exchanged with the storage unit using (5) and (8). We remark that it is possible to consider also operative and maintenance costs of the $i$ th DG unit that depend on the generated power; in this case, the term $O M_{i} P_{i}(k)$ must be added in the objective function. The term $\mathrm{OM}^{b}\left[2 z^{b}(k)-P^{b}(k)\right]$ reduces charging and discharging frequency. We recall that $C^{\mathrm{g}}(k)$ can be negative, i.e., energy is sold to the utility grid, representing an earning for the microgrid system.

In order to write the cost functional in a more compact form, we introduce, for each time $k$, the auxiliary variable $\sigma_{i}(k)$, which accounts for approximation of the $i$ th DG unit generation costs, and the vector $\mathbf{z}(k)$, which collects all the auxiliary variables as follows:

$$
\mathbf{z}(k)=\left[\begin{array}{lllll}
\boldsymbol{\sigma}^{\prime}(k) & C^{g}(k) & \mathbf{S U}^{\prime}(k) & \mathbf{S D ^ { \prime }}(k) & z^{b}(k)
\end{array}\right]^{\prime} \in \mathbb{R}^{3 N_{g}+2}
$$

where $\boldsymbol{\sigma}(k), \mathbf{S U}(k)$, and $\mathbf{S D}(k)$ are column vectors containing, respectively, all the $\sigma_{i}(k)$, the generators startup and shutdown costs, respectively. In addition, we denote by $\mathbf{u}_{k}^{T-1}$ the input sequence $\mathbf{u}_{k}^{T-1}=(\mathbf{u}(k), \ldots, \mathbf{u}(k+T-1))$ designed at time $k$, where $\mathbf{u}(k)$ has been introduced in (8).

Then, the cost functional can be rewritten as

$$
\sum_{k=0}^{T-1}\left[\mathbf{c}_{\mathbf{u}}{ }^{\prime}(k) \mathbf{u}(k)-\mathrm{OM}^{b} \mathbf{F}^{\prime}(k) \mathbf{u}(k)-\mathrm{OM}^{b} \mathbf{f}^{\prime} \mathbf{w}(k)+\mathbf{c}_{\mathbf{z}}{ }^{\prime} \mathbf{z}(k)\right]
$$

where the term $-\mathrm{OM}^{b} \mathbf{F}(k) \mathbf{u}(k)-\mathrm{OM}^{b} \mathbf{f}^{\prime} \mathbf{w}(k)$ is derived from the term $-\mathrm{OM}^{b} P^{b}(k)$ in (15), with $P^{b}(k)$ given by (8); the column vectors $\mathbf{c}_{\mathbf{z}}$ and $\mathbf{c}_{\mathbf{u}}$ are given in Appendix A.

\section{Capacity and Terminal Constraints}

To pose the final MILP optimization problem, additional operational constraints must be met

$$
\begin{aligned}
x_{\min }^{b} & \leq x^{b}(k) \leq x_{\max }^{b} \\
P_{i, \text { min }} \delta_{i}(k) & \leq P_{i}(k) \leq P_{i, \max } \delta_{i}(k) \\
\left|P_{i}(k+1)-P_{i}(k)\right| & \leq R_{i, \max } \delta_{i}(k) \\
\beta_{h, \text { min }} & \leq \beta_{h}(k) \leq \beta_{h, \text { max }}
\end{aligned}
$$

with $i=1, \ldots, N_{g}$ and $h=1, \ldots, N_{c}$. The constraints above model the physical bounds on the storage device (16a), the power flow limits of the DG units (16b) and their ramp up and ramp down rates (16c), the bounds on controllable loads curtailments (16d).

Note that the binary variable $\delta_{i}(k)$ will be equal to 1 if the power $P_{i}(k)$ generated from the $i$ th DG unit at time $k$ is strictly positive and equal to 0 if $P_{i}(k)=0$. When $P_{i, \min }=0$, a wrong 
assignment of the $\delta_{i}(k)$ variable in the inequality (16b) can be avoided by assigning to $P_{i, \min }$ a very small positive value.

\section{Model Predictive Control Problem}

In this section, we formulate the MPC optimization problem whose solution yields a trajectory of inputs and states into the future that satisfy the dynamics and constraints of microgrid operations while optimizing some given criteria. In terms of microgrid control, this means that, at the current point in time, an optimal plan is formulated (usually for the $24 \mathrm{~h}$ ) based on predictions of the upcoming demand, production from renewable energy units and energy prices. Only the first sample of the input sequence is implemented, and subsequently the horizon is shifted. At the next sampling time, the new state of the system is measured or estimated, and a new optimization problem is solved using this new information. By this receding horizon approach, the new optimal plan can potentially compensate for any disturbance that has meanwhile acted on the system. In order to present the MPC policy, we denote by $x^{b}(k+j \mid k)$, with $j>0$, the state at time step $k+j$ predicted at time $k$ employing the storage model (9).

At each time step $k$, given an initial storage state $x_{k}^{b}$ and a time duration $T$, the MPC scheme computes the optimal control sequence $\mathbf{u}_{k}^{T-1}$ solving the following finite-horizon optimal control problem:

$$
\begin{aligned}
J\left(x_{k}^{b}\right)=\min _{\mathbf{u}_{k}^{T-1}} \sum_{j=0}^{T-1} & {\left[\mathbf{c}_{\mathbf{u}}{ }^{\prime}(k+j) \mathbf{u}(k+j)+\mathbf{c}_{\mathbf{z}}{ }^{\prime} \mathbf{z}(k+j)\right.} \\
& \left.-\mathrm{OM}^{b} \mathbf{F}^{\prime}(k+j) \mathbf{u}(k+j)-\mathrm{OM}^{b} \mathbf{f}^{\prime} \mathbf{w}(k+j)\right]
\end{aligned}
$$

subject to

storage model (9) in the variable $x^{b}(\cdot \mid k)$;

constraints (10), (11), (13);

constraints (16);

$$
\begin{aligned}
& S_{i} \cdot P_{i}(k+j)+s_{i} \leq \sigma_{i}(k+j) ; i=1 \ldots N_{g} \\
& x^{b}(k \mid k)=x^{b}(k)
\end{aligned}
$$

where $S_{i}$ and $s_{i}$ are defined in (14). We recall that the vector of disturbances profiles, $\mathbf{w}(k+j)$, is assumed to be known over the prediction horizon, for $j=0, \ldots, T-1$; thus, the term $\mathrm{OM}^{b} \mathbf{f}^{\prime} \mathbf{w}(k+j)$ in the objective function does not affect the optimal solution.

According to the receding horizon strategy, only the first element of the optimal sequence $\mathbf{u}(k)$ is applied. The optimization problem (17) is repeated at time $k+1$, with the new measured/estimated state $x_{k+1 \mid k+1}^{b}=x_{k+1}^{b}$. By doing so, a feedback policy is designed.

Note that in the MPC scheme, applied in this paper, the controller makes its control decision by assuming that the predictions are correct (i.e., certainty equivalence).

\section{E. Initial Conditions}

At each time $k$, the system model is initialized to the measured/estimated current state of the microgrid components, i.e., the storage current energy level, loads, ON-/OFF-state and power levels generated by the noncontrollable generation units.

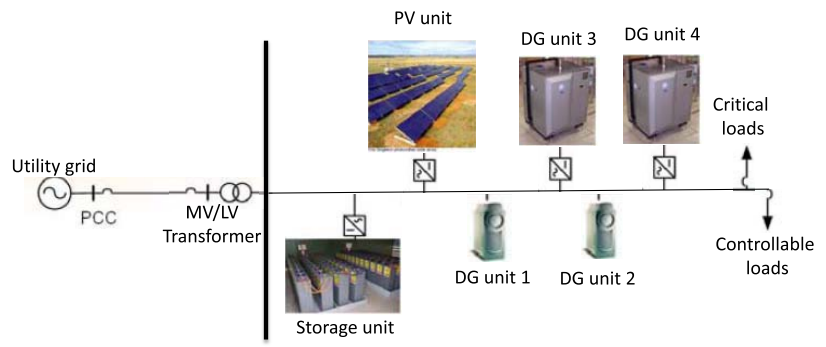

Fig. 1. Scheme of microgrid considered in simulations.

TABLE IV

GENERATOR PARAMETERS

\begin{tabular}{|l|l|l|l|l|l|}
\hline DG unit & $P_{\min }$ & $P_{\max }$ & $a_{1}$ & $a_{2}$ & $a_{3}$ \\
\hline Unit 1 & 6 & 50 & 0.0013 & 0.062 & 1.34 \\
Unit 2 & 16.4 & 92 & 0.001 & 0.057 & 1.14 \\
Unit 3 & 16 & 90 & 0.0004 & 0.06 & 1.14 \\
Unit 4 & 12.3 & 72 & 0.0006 & 0.058 & 1.9 \\
\hline
\end{tabular}

\section{F. Solving the Optimization Problem}

The MPC optimization problem is a MILP problem. The branch-and-bound techniques are mostly applied to MILP problems [41], [42]. The main advantage of the branch and bound method is that if a solution is reached, the solution is known to be globally optimal.

\section{G. Implementation Details}

The formulation presented in Section III-F was implemented using MATLAB. We used ILOG's CPLEX 12.0 [43] (an efficient solver based on the branch-and-bound algorithm) to solve the MILP optimizations. All computations are done on an Intel Core 2 Duo CPU, $2 \mathrm{GHz}$.

\section{Simulation Setup}

The microgrid we consider in the simulations is shown in Fig. 1; it is in a grid-connected mode and comprises PV panels with maximum power of $16 \mathrm{~kW}$ and four DG units. An energy storage is included, bounded between 25 and $250 \mathrm{kWh}$ and with maximal charge and discharge rates, respectively, 150 and $-150 \mathrm{~kW}$. The charge and discharge efficiencies are both equal to 0.9. Table IV describes the DG units parameters, based on data provided in [38] and [44]. The microgrid is connected to the utility grid, so power can be bought or sold. The daily spot prices (from the EEX, European Energy Exchange, on a certain day) are shown in Fig. 2. For simulation study, we chose a sampling time of $1 \mathrm{~h}$ and a prediction horizon of $24 \mathrm{~h}$. Simulations are performed over one day.

\section{A. Control Strategies Comparison}

We compare the following strategies for the microgrid optimization problem.

1) Heuristic: This is the heuristic algorithm described below in Section IV-A1.

2) MILP: It is the open loop solution obtained by solving a single MILP problem. 


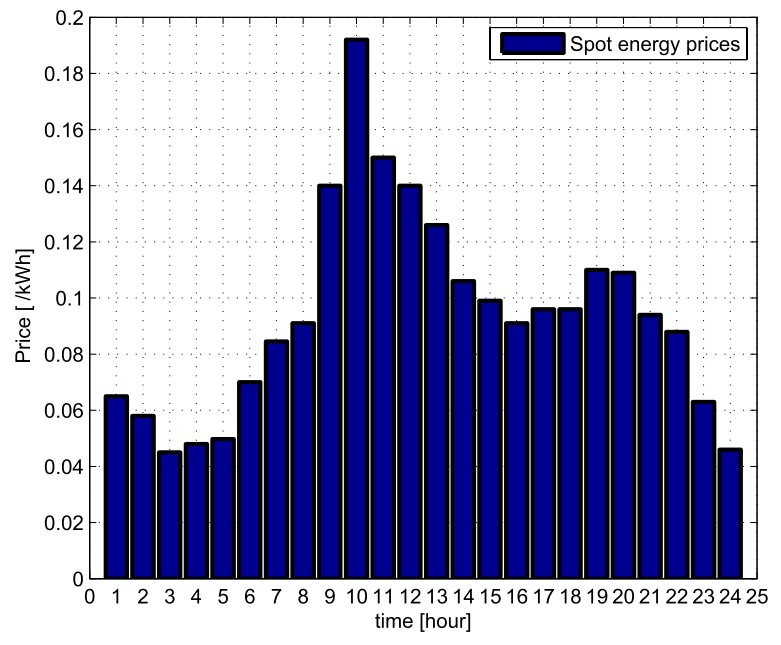

Fig. 2. Spot energy prices over $24 \mathrm{~h}$.

3) MPC-MILP: It is the feedback control law computed through the MPC control scheme. As said before, in MPC-MILP a certainty equivalence approach is applied, meaning that the predictions are assumed to be perfect in the MPC problem, i.e., unaffected by errors. The uncertainty is then compensated by the feedback mechanism.

4) Benchmark: The 24-h horizon plan obtained by solving the optimization problem assuming no errors in forecasts, which means that the scheduler knows all the actual values for loads and RES generation over the whole prediction horizon. The benchmark can never be achieved but it provides an ideal value to evaluate a strategy against.

When the MILP and MPC-MILP strategies are applied, we also include in the strategy evaluation the cost of purchasing the necessary amount of energy from the utility grid in case there is demand surplus due to wrong predictions of load and RES power generation. We further consider the 24-h horizon plans obtained when a $250-\mathrm{kWh}$ storage is employed.

1) Heuristic Algorithm: We had in mind to compare the proposed approach with a heuristic microgrid management algorithm but, to the best of our knowledge, none has been proposed in the literature so far, nor are we aware of practical approaches to the problem, beyond straightforward balancing of supplied and demanded powers, which takes into no account the economical side of the problem. Hence, we propose one here.

For simplicity's sake, we assume no maintenance, startup and shutdown costs. In addition, no storage unit is utilized, so that dynamics have not to be accounted for. The loads are all critical loads. By doing so, the possible combinations of power inputs can be more easily handled (also by a human operator); namely, the microgrid operator can decide about the DG unit generation and the interaction with the utility grid. In addition to that the minimum up and down times are assumed to be equal to 1 sampling time. The capacity constraints are considered, although we assume that the bilateral contract between the microgrid and the utility grid will ensure the energy balance to be always achievable, meaning that the microgrid operator will be always allowed to purchase from or

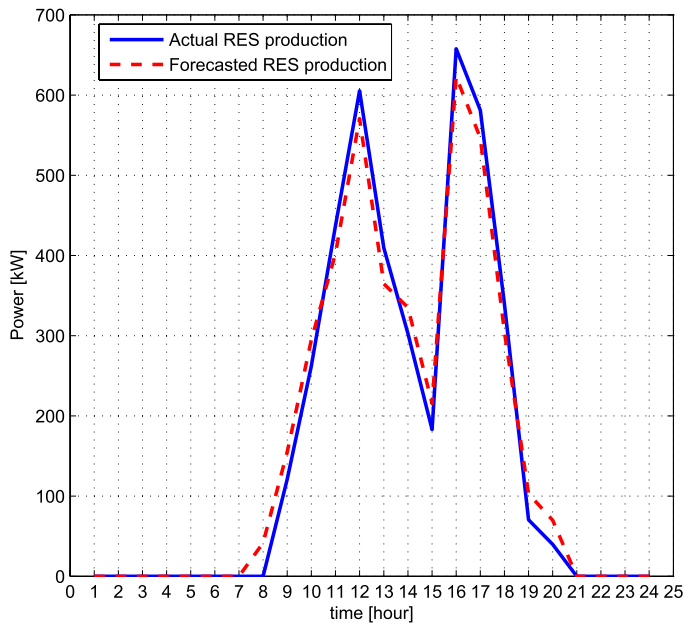

Fig. 3. Forecasted and actual PV power generation over $24 \mathrm{~h}$.

sell to the utility grid the necessary amount of energy. Finally, we assume that a turned-on DG unit will be always generating the maximum available power.

The heuristic algorithm consists of three steps, applied at each sampling time; these steps are illustrated in Appendix B.

\section{B. Forecasts}

In order to apply the control strategy described in Section III, renewable power and demand forecasts have to be computed. The renewable power and demand data series generally exhibit high-frequency fluctuations and peak shifting, also influenced by meteorological factors, such as outside temperature and irradiance. Therefore, it is a relatively hard task to capture the dynamics of these series and fit a model out of the given data set. Broadly exploited methodologies for nonlinear forecasting are neural networks (NNs) [45] and support vector machines (SVMs). The latter is a powerful statistical method aiming at capturing the underlying structure in a data set based on input training data [46], [47]; recently, the SVM technique is being successfully applied to demand forecasting and for predicting renewable generation [48], [49].

In this paper, we apply least-square SVMs for regression (support vector regression) with a moving time window to forecast the renewable power generation and the demand for the day ahead. See Appendix C for an outline of the algorithm and further details on forecasting in simulations and experiments.

Examples of renewable power production profiles and daily demand employed in the optimization routine are shown, respectively, in Figs. 3 and 4.

\section{Simulation Result}

Figs. 5 and 6 show, respectively, the power exchanged with the utility grid and the DG unit power generation obtained by applying the different strategies without storage. It is shown that the MPC-MILP leads to a more efficient utilization of the DG units and a larger amount of energy is sold to the utility grid. 


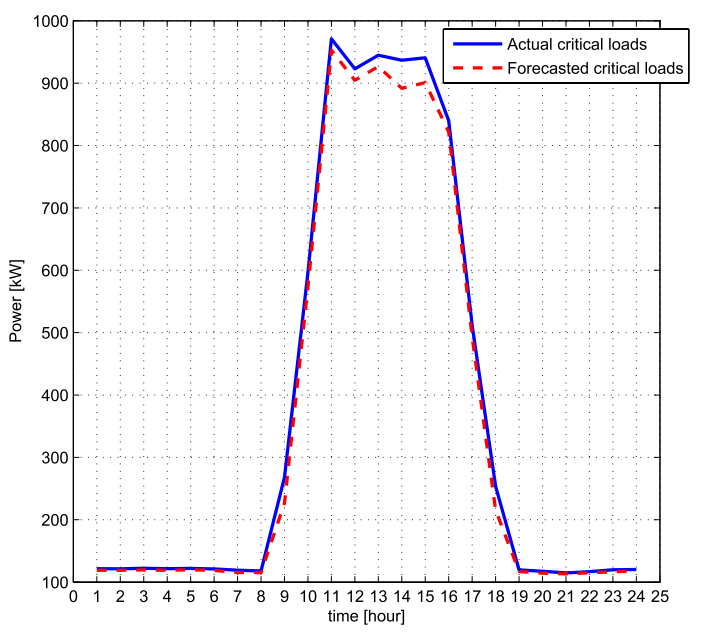

Fig. 4. Forecasted and actual demand over $24 \mathrm{~h}$.
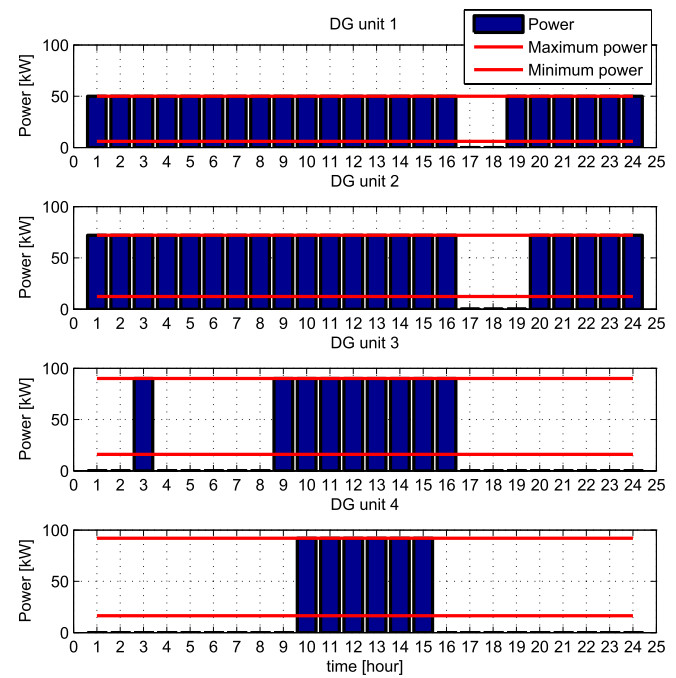

(a)
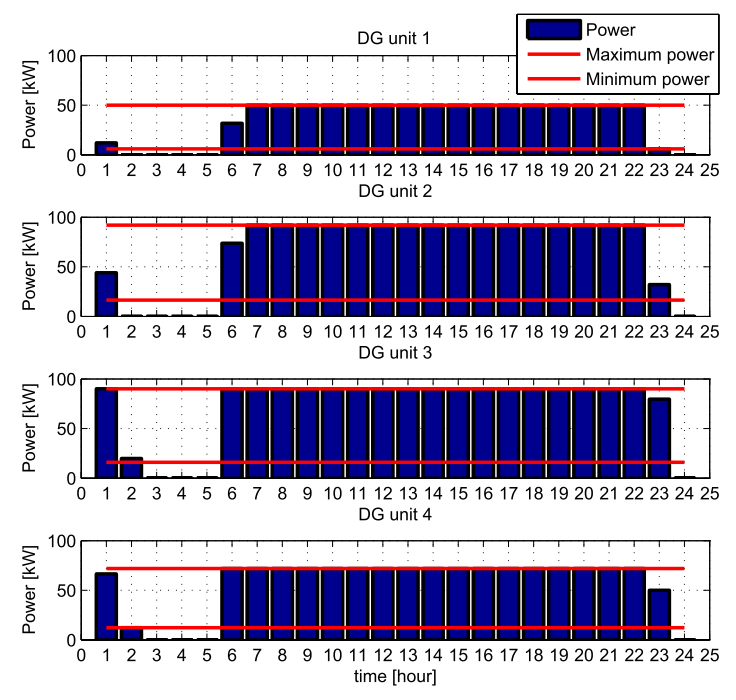

(b)

Fig. 5. DG units power generation over $24 \mathrm{~h}$. (a) Heuristic. (b) MPC-MILP.

Table $\mathrm{V}$ reports the performance of the described strategies for microgrid operation optimization without storage utilization. It shows that the cost increment with respect to the

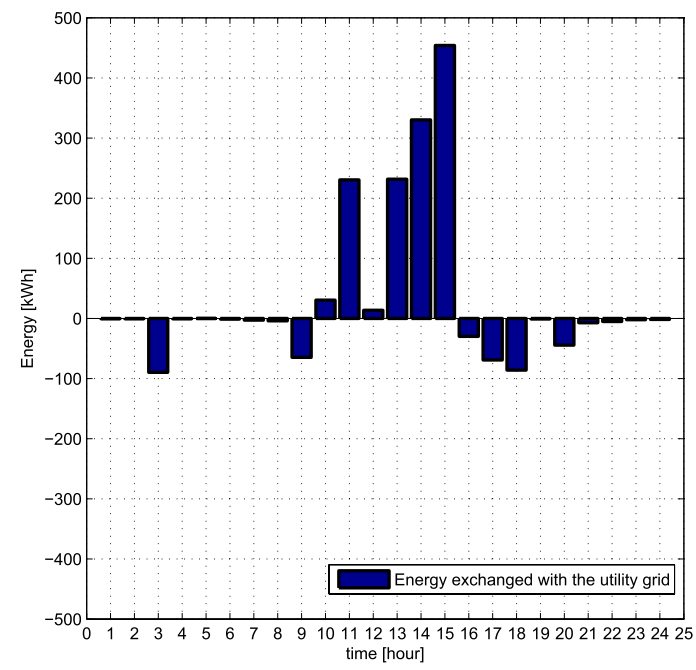

(a)

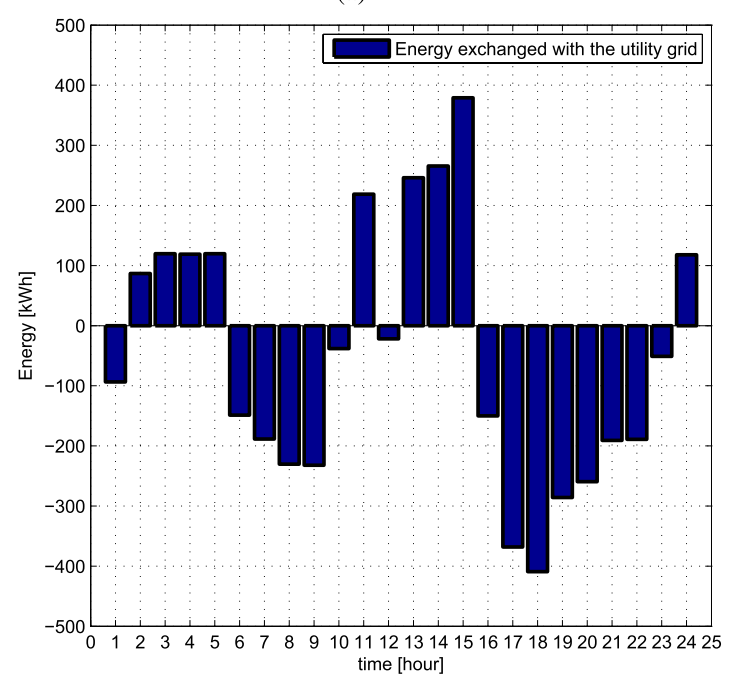

(b)

Fig. 6. Purchased/sold energy over 24 h. (a) Heuristic. (b) MPC-MILP.

TABLE V

COMPARISON OF MICROGRID OPERATION OPTIMIZATION Strategies Without Storage

\begin{tabular}{|l|l|l|}
\hline Strategy & Total cost $(€)$ & Correction costs $(€)$ \\
\hline Heuristic & 452.8 & 0 \\
MPC-MILP & 418.9 & 59.5 \\
Benchmark & 416.7 & 0 \\
\hline
\end{tabular}

benchmark is $8.6 \%$ for the heuristic algorithm and only $0.5 \%$ for the proposed algorithm.

Consider now the possibility to employ the $250-\mathrm{kWh}$ storage unit. Fig. 7 shows the storage utilization during the planning horizon. Table VI shows that the storage makes the microgrid more economically efficient. It is also reported that MILP without feedback action yields poor results, due to the uncertainties in the demand and in the PV power generation. Fig. 8 shows that the MPC-MILP strategy yields less violations of the storage capacity constraints with respect to MILP; the figure also shows that the MPC-MILP strategy produces in both cases a solution close to the benchmark. As the storage level cannot be less than the storage minimum capacity, the 


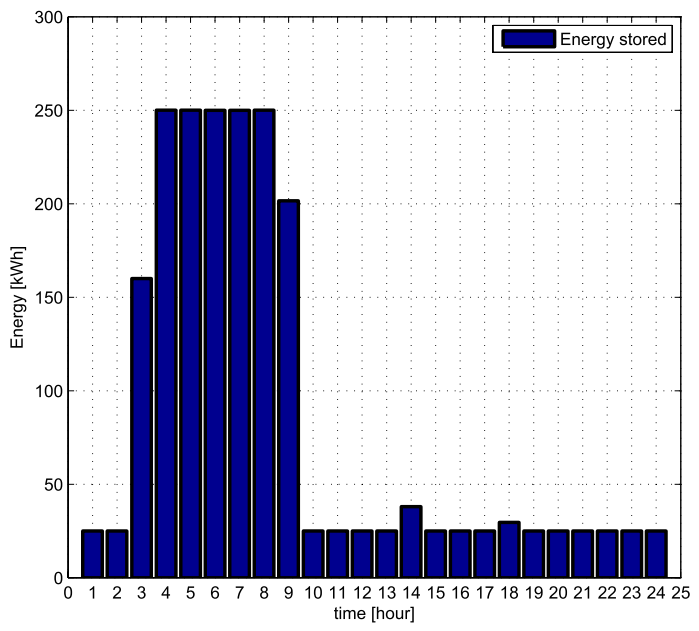

Fig. 7. Energy stored over $24 \mathrm{~h}$.

TABLE VI

COMPARISON OF MICROGRID OPERATION OPTIMIZATION Strategies With StORAGE

\begin{tabular}{|l|l|l|}
\hline Strategy & Total cost $(€)$ & Correction costs $(€)$ \\
\hline MPC-MILP with storage & 403.3 & 49.8 \\
MILP with storage & 788.3 & 435.8 \\
Benchmark with storage & 391.5 & 0 \\
\hline
\end{tabular}

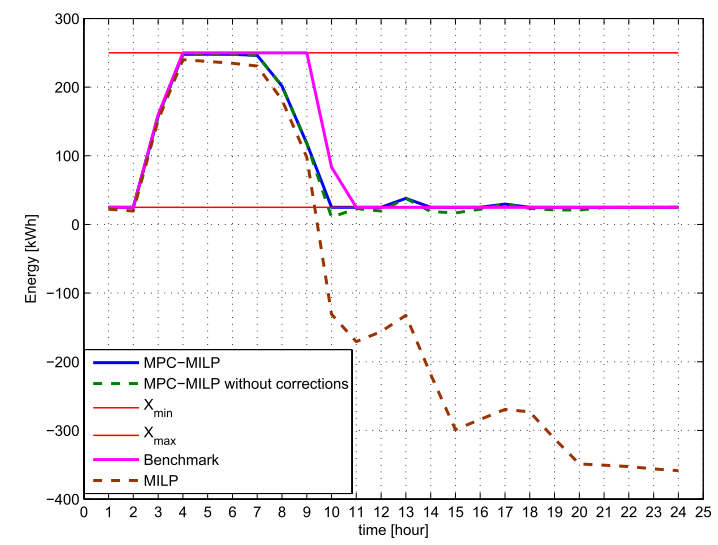

Fig. 8. Usage of storage under different strategies (negative values mean the storage would lend energy, not possible in the real case).

correction action is to buy from the utility grid the amount of energy needed to lead the storage level to its minimum capacity value.

\section{A. Computational Complexity}

It is well known that MILP problems are NP complete and their computational complexity mainly depends on the number of integer variables [39], [50]. We investigated the computational burden of the MILP optimization problems to be solved online, in order to assess the feasibility of the proposed approach. We considered the case study reported above, by using different prediction horizons $T$. At each time instant, an MILP problem is solved. Table VII reports the computation times (average and worst case) needed for solving the MILPs as well as the problem sizes as the prediction horizon grows. The computation times increase as the prediction horizon $T$
TABLE VII

COMPUTATION Times

\begin{tabular}{|l|l|l|l|l|}
\hline Horizon & $\begin{array}{l}\text { Number of } \\
\text { decision variables }\end{array}$ & $\begin{array}{l}\text { Number of } \\
\text { constraints }\end{array}$ & $\begin{array}{l}\text { Average } \\
\text { times [s] }\end{array}$ & $\begin{array}{l}\text { Worst case } \\
\text { times [s] }\end{array}$ \\
\hline 24 & 576 & 3561 & 3.3 & 6.0 \\
36 & 864 & 5397 & 5.5 & 7.4 \\
48 & 1152 & 7234 & 9.1 & 12.2 \\
60 & 1440 & 9070 & 12.6 & 19.3 \\
72 & 1728 & 10907 & 17.7 & 24.3 \\
\hline
\end{tabular}

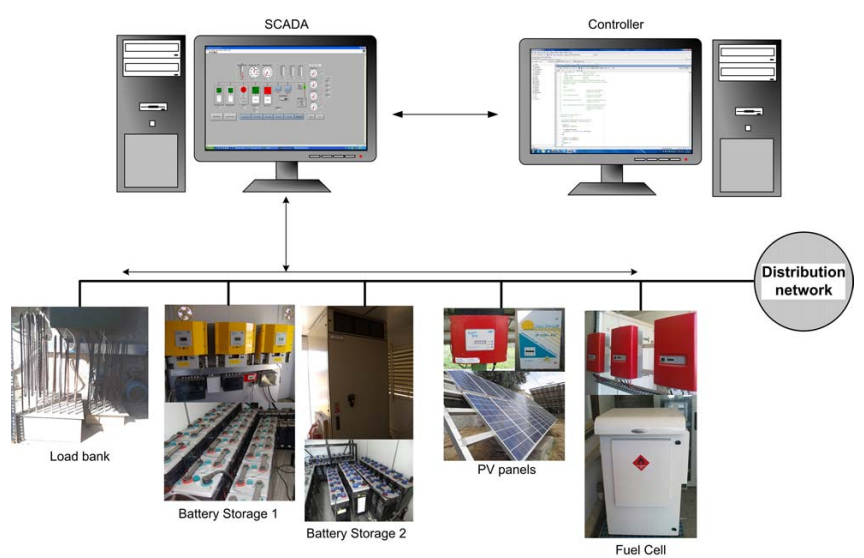

Fig. 9. Experimental microgrid setup.

becomes longer. However, the solution to the optimization problem took at most $24.3 \mathrm{~s}$, a time much shorter than the sampling time of $1 \mathrm{~h}$. A longer prediction horizon would not usually provide an improvement because the forecasts degrade as time increases, but Table VII shows that the computational burden can be affordable. This can also mean that the sampling time could be reduced at the cost of a negligible increase of computational effort. Actually, the experiments were run with a 15-min sampling period.

\section{EXPERIMENTAL RESULT}

The experimental validation of the control algorithm was performed at the Center for Renewable Energy Sources and Saving (CRES), Pikermi-Athens, Greece. We consider a 15-min sampling period and the experiments are run over $6 \mathrm{~h}$. The facility used for this purpose is the experimental microgrid of CRES comprising the following units (Fig. 9).

1) RES: Two PV units with maximum power 1.1 and $2.5 \mathrm{~kW}$.

2) DG units: One proton exchange membrane fuel cell of $5 \mathrm{~kW}$ and a battery storage used in order to simulate the operation of a CHP unit. The maximum power of the CHP was selected to $2 \mathrm{~kW}$. The operative and maintenance costs of the fuel cell are $0.16 €$ per sampling period $(15 \mathrm{~min})$. The operative and maintenance costs of the CHP are $0.01 €$ per $\mathrm{kW}$ generated.

3) Storage unit: One battery storage system with $40-\mathrm{kWh}$ maximum capacity and $2.5-\mathrm{kW}$ maximum power. The charge and discharge efficiencies are both equal to 0.8 . We assume that the battery never discharges below $75 \%$ of its maximum capacity. The operative and maintenance costs of the battery are $0.0784 €$ per $\mathrm{kWh}$ exchanged without associated inverter costs. 


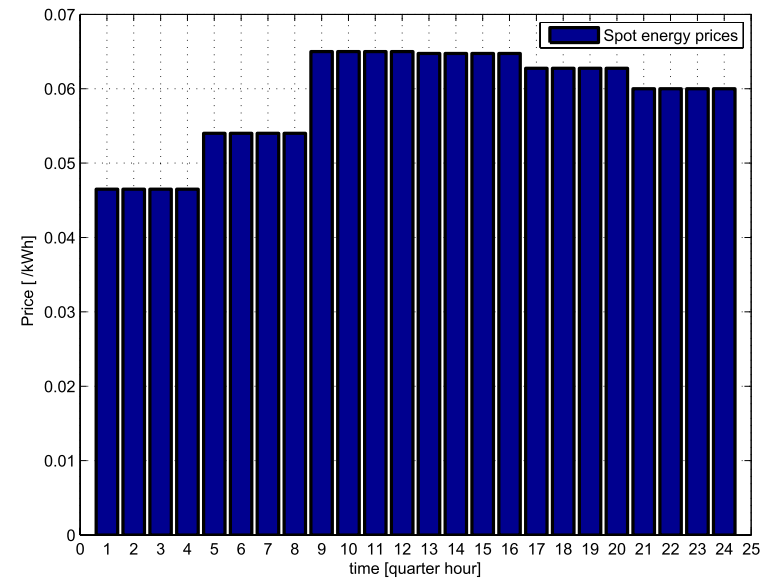

Fig. 10. Spot energy prices over 24 time steps $(6 \mathrm{~h})$ (all experiments).

4) Loads: A bank of resistive loads was used. Specifically, the loads were divided into two groups (critical and flexible) each of which was programmed through a specific load curve with 10-min sampling time. According to the control set points, an amount of the controllable load was curtailed while the maximum load consumption was about $9 \mathrm{~kW}$.

All units were interconnected through the microgrid three-phase power line to the public grid.

Low level controllers for microgrid components were available.

1) A local integrator was employed to regulate the battery inverter frequency according to the active power set point.

2) A local integrator was used for the $\mu$-CHP inverter.

3) The fuel cell unit was regulated by using a local integrator and a comparator with the mean power reference value.

4) Concerning the load bank, a local controller selected the most appropriate combination of resistors, which provided the closest consumption value according to the set point.

The control algorithm was implemented on a PC interfaced with the microgrid SCADA system through the local area network.

All data used in experiments are realistic, based on measurements, datasheets, and market prices. Loads follow a realistic profile derived from real consumption measurements in a real microgrid. The daily spot prices are shown in Fig. 10.

The results of three experiments are presented.

1) Experiment 1: The microgrid is operated without highlevel control.

2) Experiment 2: The microgrid operations are managed by the MPC-MILP control scheme with a planning horizon of 24 steps.

3) Experiment 3: The microgrid operations are managed by the MPC-MILP control scheme with a planning horizon of 72 steps.

The experiments are run from 9 A.M. to 3 P.M., so the PV units generate some power at all time steps (1 kW on average).

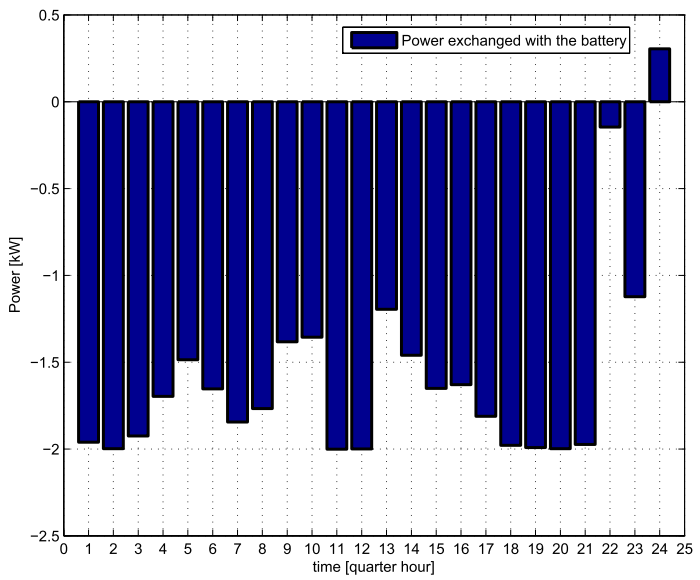

(a)

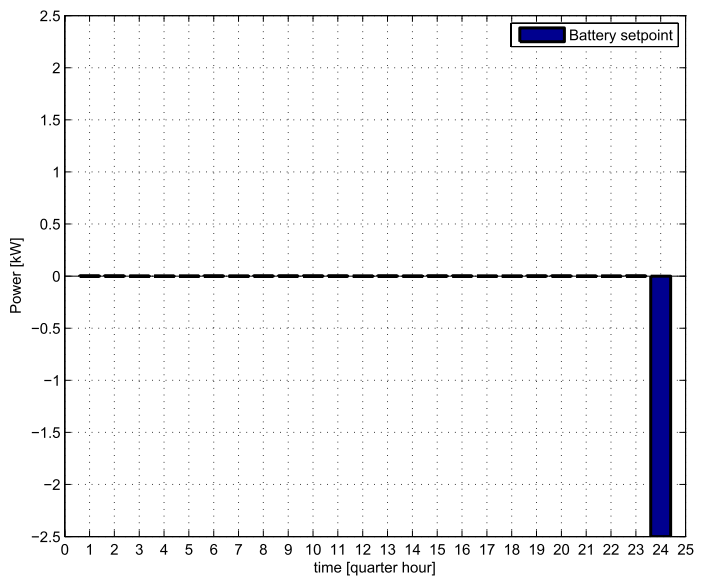

(b)

Fig. 11. Battery usage over 24 time steps (6 h). (a) Experiment 1 (no MPC). (b) Experiment 2 (MPC over 24 time steps).

During all the three experiments, the system is operated with the same consumption profiles. The ultimate purpose of Experiment 1 is the power balance between production and consumption. In other words, the system operated so as to minimize the power/energy flow to and from the public grid. This is the fundamental concept of microgrids since they are designed so as to exploit as much as possible the benefits of DERs [2].

The battery is heavily employed in Experiment 1; much less utilized in Experiment 2 and not utilized at all in Experiment 3 due to its high maintenance costs (Fig. 11). A smaller amount of power needs to be purchased from the utility grid during Experiment 1 compared with the experiments run with the high level controller (Figs. 12 and 13). Further the fuel cell is utilized in Experiment 1 (Fig. 14), while it is always OFF during the experiments with high-level control because of its large operative and maintenance costs and its low efficiency in electrical power generation. It would be likely utilized in case the thermal demand and the emissions were included in the problem formulation.

The CHP unit is always run at its maximum power in these experiments.

The total cost for Experiment 1 is $27.4 €$, for Experiment 2 is $19.6 €$, and for Experiment 3 is $17.9 €$. Then, the 


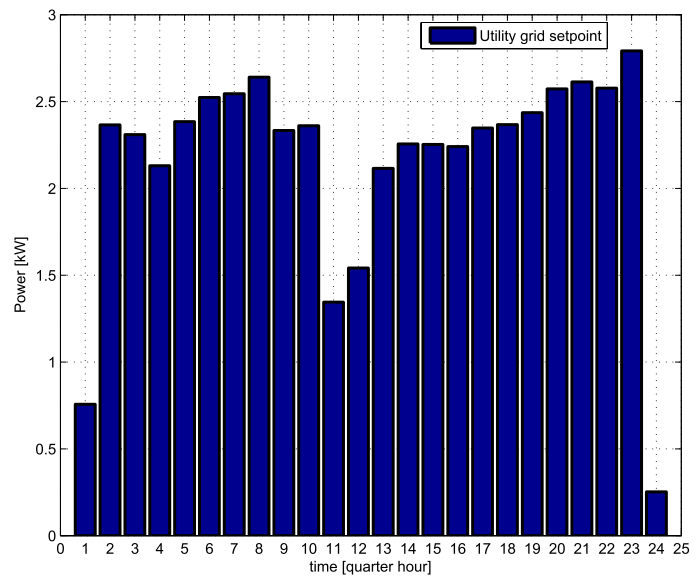

(a)

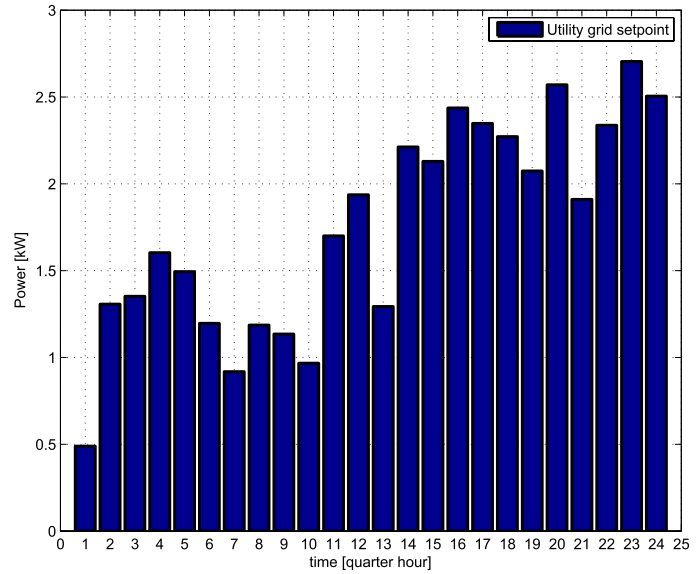

(b)

Fig. 12. Energy exchanged with the utility grid over 24 time steps $(6 \mathrm{~h})$. (a) Experiment 2 (MPC over 24 time steps). (b) Experiment 3 (MPC over 72 time step).

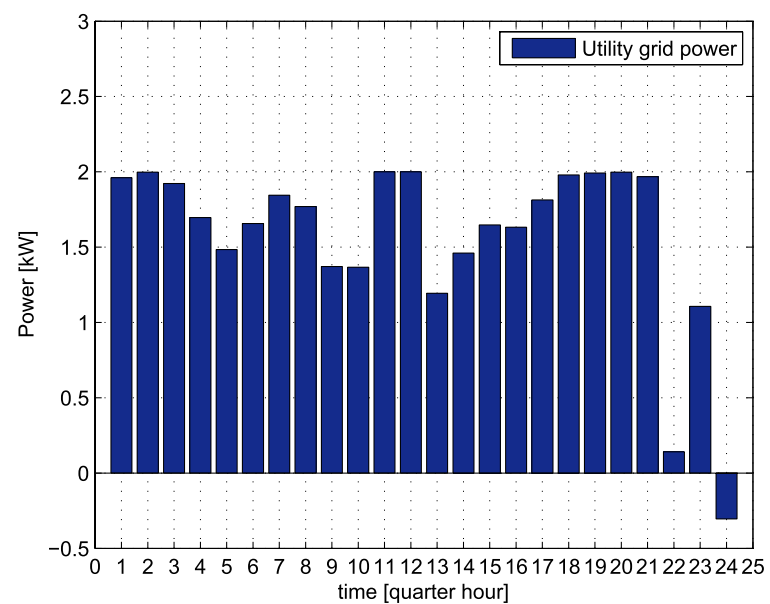

Fig. 13. Energy exchanged with the utility grid over 24 time steps $(6 \mathrm{~h})$ for Experiment 1 (no MPC).

MPC-MILP strategy yields a $28.5 \%$ saving with $T=24$ and a $34.7 \%$ saving with a longer prediction horizon, $T=72$.

Curtailments are usually penalized since they lead to user discomfort; so they are not performed unless strictly convenient or necessary. Hence, all experiments run with a penalty on curtailments $\rho_{c}$ equal to 0.5 , show no curtailment. We ran

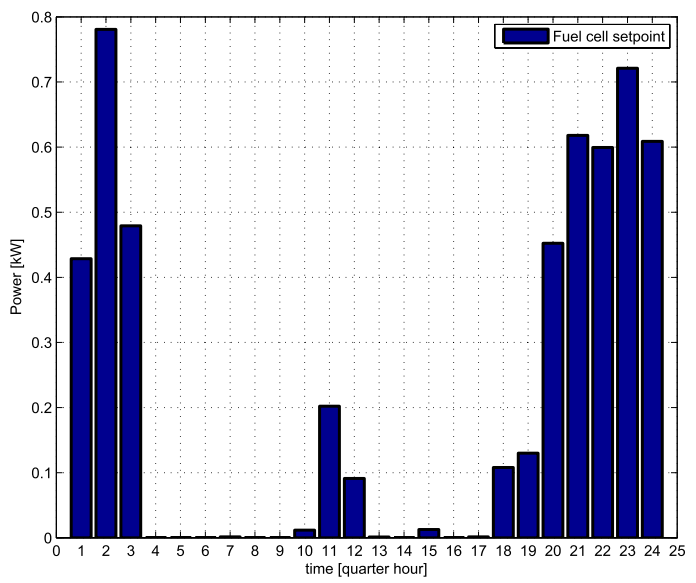

Fig. 14. Fuel cell power generation for Experiment 1 (no MPC).

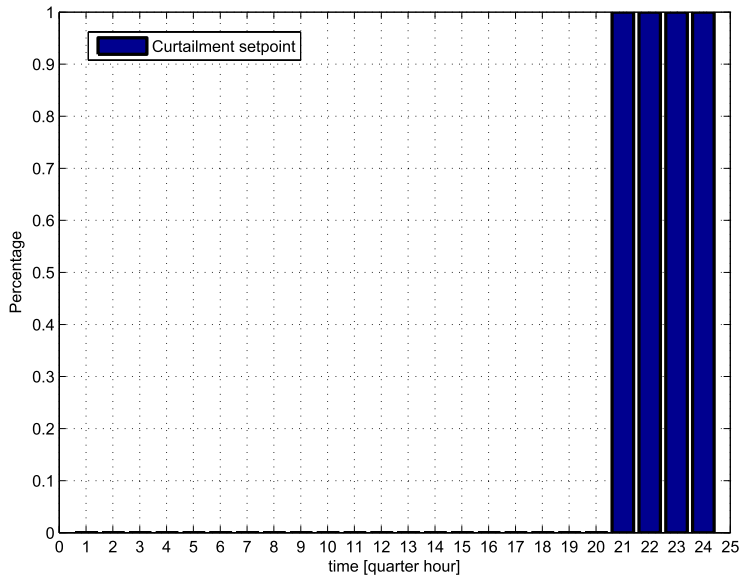

Fig. 15. Curtailments over 24 time steps (6 h).

another experiment over 24 steps reducing $\rho_{c}$ to 0.1 in the last hour and Fig. 15 shows how the optimization algorithm uses this relaxation. The economic saving with respect to the experiment with $\rho_{c}=0.5$ is not significant because the PV power generation during this experiment was much smaller, so a larger amount of energy needed to be bought from the utility grid. A tradeoff between cost and demand peak reduction and user comfort can be achieved by tuning the parameter $\rho_{c}$.

It is worth mentioning that under some circumstances the actual power values deviated from the setpoints due to the following reasons.

1) The battery storage inverter presented power derating when overheated. This led to a power reduction. As a result, the microgrid covered the deficit by absorbing power from the public grid.

2) The second battery system, which was used to simulate the CHP unit, presented at specific moments a deep discharge rate due to the bad battery state of health. Because of this, it was necessary to manually reverse the power for small intervals of $15 \mathrm{~min}$. This, despite not being counted as energy absorption, was considered as zero production intervals, leading to deviation from the setpoints. By using a better battery can sort this issue out. 
3) In some experiments, the weather conditions led to reduced power from the PV and hence to deviation from the predicted power.

\section{CONCLUSION}

In this paper, we propose a novel mixed integer linear approach on modeling and optimization of microgrids. We bring into account unit commitment, economic dispatch, energy storage, sale and purchase of energy to/from the main grid, and curtailment schedule. First, we assume perfect knowledge of the microgrid state, renewable resources production, future loads, and so on, which is useful to solve the optimization problem. Further, to cope with inevitable disturbances and forecast errors, we embed this into an MPC framework. The proposed approach was investigated on an experimental microgrid located in Athens, Greece. Experimental results show that our MPC-MILP control scheme is able to economically optimize the microgrid operations and save money compared with the current practice. The results also evidence that a tradeoff between demand peak reduction and user comfort can be achieved through allowable curtailments.

Future work will focus on relaxing our assumptions so as to include state estimation and uncertainty modeling. Finally, heat recovery capabilities and reactive power are not considered in the microgrid modeling and problem formulation to limit its complexity. We are aware of their importance and their incorporation into the proposed control framework is under current study.

$$
\begin{aligned}
& \text { APPENDiX A } \\
& \text { MATRICES }
\end{aligned}
$$

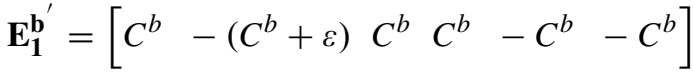

$$
\begin{aligned}
& \mathbf{E}_{\mathbf{2}}^{\mathbf{b}^{\prime}}=\mathbf{E}_{\mathbf{2}}^{\mathbf{g}^{\prime}}=\left[\begin{array}{llllll}
0 & 0 & 1 & -1 & 1 & -1
\end{array}\right] \\
& \mathbf{E}_{\mathbf{3}}^{\mathbf{b}^{\prime}}=\left[\begin{array}{llllll}
1 & -1 & 1 & -1 & 0 & 0
\end{array}\right] \\
& \mathbf{E}_{\mathbf{4}}^{\mathbf{b}^{\prime}}=\left[\begin{array}{llllll}
C^{b} & -\varepsilon & C^{b} & C^{b} & 0 & 0
\end{array}\right] \\
& \mathbf{E}_{\mathbf{1}}^{\mathbf{g}^{\prime}}=\left[\begin{array}{llllll}
T^{g} & -\left(T^{g}+\varepsilon\right) & M^{g} & M^{g}-M^{g} & -M^{g}
\end{array}\right] \\
& \mathbf{E}_{\mathbf{3}}^{\mathbf{g}^{\prime}}(k)=\left[\begin{array}{llllll}
1 & -1 & c^{P}(k) & -c^{P}(k) & c^{S}(k) & -c^{S}(k)
\end{array}\right] \\
& \mathbf{E}_{\mathbf{4}}^{\mathbf{g}^{\prime}}=\left[\begin{array}{llllll}
T^{g} & -\varepsilon & M^{g} & M^{g} & 0 & 0
\end{array}\right]
\end{aligned}
$$

where $M^{g}=\max _{k}\left(c^{P}(k), c^{S}(k)\right) \cdot T^{g}, \varepsilon$ is a small tolerance (typically the machine precision)

$$
\begin{aligned}
& \mathbf{F}^{\prime}(k)=[\underbrace{1 \ldots 1}_{N g} 1 \underbrace{\ldots D_{i}^{c}(k) \ldots}_{N c} \underbrace{0 \ldots 0}_{N g}] \\
& \mathbf{f}^{\prime}=[1 \underbrace{-1 \cdots-1}_{N l} \underbrace{-1 \cdots-1}_{N c}] \\
& \mathbf{c}_{\mathbf{z}}^{\prime}=\left[\begin{array}{lll}
\underbrace{1 \ldots 1}_{N g} 1 \underbrace{1 \ldots 1}_{2 \cdot N g} 2 \cdot \mathrm{OM}^{b}
\end{array}\right] \\
& \mathbf{c}_{\mathbf{u}}(k)^{\prime}=[\underbrace{0 \ldots 0}_{N g} 1 \underbrace{\ldots \rho_{i}(k) D_{i}^{c}(k) \ldots}_{N c} \underbrace{\ldots O M_{i} \ldots}_{N g}] .
\end{aligned}
$$

\section{APPENDIX B}

\section{Steps of the Heuristic Algorithm}

The heuristic algorithm consists of the following steps.

1) The difference between the power generated from all the RES units and the actual load is computed. The power generated from the renewable energy sources is then always utilized to satisfy the demand. If the difference is positive, the power surplus is sold to the utility grid, the time step is incremented by one and the algorithm goes back to step 1; otherwise it proceeds to step 2 .

2) If the cost to purchase the demand deficit from the utility grid is smaller than the minimum cost among the DG unit generating costs, the deficit will be filled by purchasing the corresponding amount of energy from the utility grid, the time step is incremented by one and the algorithm goes back to step 1; otherwise it proceeds to step 3.

3) The DG units are turned on from the cheapest to the most expensive one until the demand surplus is covered. Since the DG units are run at their maximum power capacity, it is likely to have power surplus once the total demand is met; then the corresponding energy is sold to the utility grid; the time step is incremented by one and the algorithm goes back to step 1.

\section{APPENDix C \\ BACKGROUND}

In this section, we briefly introduce some basic concepts employed in the control strategy design.

\section{A. Mixed Integer Linear Programming}

In a microgrid system continuous and discrete-valued dynamics interact. The physical quantities, such as energy and power flows, can be represented by continuous variables, while the discrete features of microgrid components (e.g., the ON/OFF status of the DGs, the storage charging/discharging state, and the minimum up and down time constraints) can be captured by using binary decision variables. In addition, the behavior of a microgrid system and its components can be described by both differential or difference equations (e.g., storage dynamics) and logical statements, i.e., statements of the form if ...then ...else. Since we are interested in model predictive control, we need to construct a prediction model of the system. In [36], it is shown how to cast a logical statement of a given form into linear mixed-integer constraints, i.e., constraints involving both continuous and discrete-valued variables. In the following, we will provide some example, taken from [36], of equivalences between logical statements and linear mixed-integer constraints; the statement

$$
f(k) \geq 0 \Longleftrightarrow \delta=1
$$

is true if and only if $\begin{cases}-m \delta & \leq f(k)-m \\ -(M+\varepsilon) \delta & \leq-f(k)-\varepsilon\end{cases}$ 
similarly

$$
y=\delta f(k) \text { is equivalent to }\left\{\begin{array}{l}
y \leq M \delta \\
y \geq m \delta \\
y \leq f(k)-m(1-\delta) \\
y \geq f(k)-M(1-\delta)
\end{array}\right.
$$

where $f$ is a function upper and lower bounded by $M$ and $m$, respectively, $\delta$ is a binary variable, $y$ is a real variable, and $\varepsilon$ is a small tolerance (typically the machine precision). The tolerance $\varepsilon$ is needed to transform a constraint of the form $y<0$ into $y \leq 0$, since MILP solving algorithms only handle nonstrict inequalities.

\section{B. SVR Training Algorithm}

The training algorithm of a SVR involves a quadratic optimization program, which provides a unique solution and does not require the random initialization of weights, as in training of NNs. The training data set is defined as follows:

$$
\left\{x_{i}, y_{i}\right\}, \quad i=1, \ldots, N
$$

where $N$ is the number of samples, $x_{i}, y_{i}$ are real-valued input patterns and corresponding outputs, respectively. The SVR training algorithm aims at finding a nonlinear mapping $\phi(x)$ of the input data $x$ and then solving a linear regression problem in this feature space. The function representing the relationship between the output and input is

$$
y_{i}=\sum_{i=1}^{N} w_{i} \phi\left(x_{i}\right)+b_{i}
$$

where for each sample $i, b_{i}$ is the scalar thresholds and $w_{i}$ is the weight coefficient. Then, the parameters $w_{i}$ and $b_{i}$ are estimated by solving the following convex regression problem in this feature space:

$$
\min _{w, b, \xi, \xi^{*}} 0.5 w^{T} w+C \sum_{i=1}^{N}\left(\xi+\xi^{*}\right)
$$

s.t.

$$
\begin{aligned}
& y_{i}-w^{T} \phi\left(x_{i}\right)-b_{i} \leq \epsilon+\xi^{*} \\
& w^{T} \phi\left(x_{i}\right)+b_{i}-y_{i} \leq \epsilon+\xi \\
& \xi, \xi^{*} \geq 0, \quad i=1 \ldots N
\end{aligned}
$$

where the parameter $C$ is the regularization parameter, which assigns penalty to the errors and determines a tradeoff between the flatness of the regression function and the training error, $\xi$ and $\xi^{*}$ are the slack variables of the upper and the lower bound of the training vector, and $\epsilon$ is the residual tolerance.

All the forecasts are obtained by MATLABs SVM toolbox, a LS-SVM training and simulation environment written in C-code [51]. Patterns are updated by adding the most recent actual value and the oldest value is dropped, and the forecasts for the next $T$ sampling periods are computed.

\section{ACKNOWLEDGMENT}

The authors would like to thank the Department of PVs and DER systems, Center for Renewable Energy Sources and Saving for the support provided during the experiments.

\section{REFERENCES}

[1] R. Lasseter and P. Piagi, "Microgrid: A conceptual solution," in Proc. IEEE Annu. Power Electron Specialists Conf., Jun. 2004, pp. 4285-4290.

[2] N. Hatziargyriou, H. Asano, R. Iravani, and C. Marnay, "Microgrids," IEEE Power Energy Mag., vol. 5, no. 4, pp. 78-94, Jul./Aug. 2007.

[3] T. Ustun, C. Ozansoy, and A. Zayegh, "Recent developments in microgrids and example cases around the world. a review," Renew. Sustain. Energy Rev., vol. 15, no. 8, pp. 4030-4041, 2011.

[4] (2008). Strategic Deployment Document for Europe's Electricity Networks of the Future [Online]. Available: http://www.smartgrids.eu/

[5] J. Ilic, M. Prica, S. Rabiei, J. Goellner, D. Wilson, C. Shih, et al., "Technical and economic analysis of various power generation resources coupled with CAES systems," Nat. Energy Technol. Lab., Morgantown, WV, USA, Tech. Rep. DOE/NETL-2011/1472, Jun. 2011.

[6] M. Hoffman, M. Kintner-Meyer, A. Sadovsky, and J. DeSteese, "Analysis tools for sizing and placement of energy storage for grid applications-A literature review," Pacific Northwest Nat. Lab. (DOE/PNNL-19703), Richland, WA, USA, Tech. Rep., Sep. 2010.

[7] Y. Chen, S. Lu, Y. Chang, T. Lee, and M. Huc, "Economic analysis and optimal energy management models for microgrid systems: A case study in Taiwan," Appl. Energy, vol. 103, pp. 145-154, Mar. 2013.

[8] M. Marzband, A. Sumper, J. Dominguez-Garcia, and R. Gumara-Ferret, "Experimental validation of a real time energy management system for microgrids in islanded mode using a local day-ahead electricity market and MINLP," Energy Convers. Manag., vol. 76, pp. 314-322, Dec. 2013.

[9] Z. Dinghuan, R. Yang, and G. Hug-Glanzmann, "Managing microgrids with intermittent resources: A two-layer multi-step optimal control approach," in Proc. NAPS, Sep./Oct. 2010, pp. 1-8.

[10] R. O’Neill, T. Dautel, and E. Krall, "Recent ISO software enhancements and future software and modeling plans," Staff Report, Federal Energy Regulatory Commission, Washington, DC, USA, Tech. Rep., Nov. 2011.

[11] R. Sioshansi, R. O'Neill, and S. Oren, "Economic consequences of alternative solution methods for centralized unit commitment in dayahead electricity markets," IEEE Trans. Power Syst., vol. 23, no. 2, pp. 344-352, May 2008.

[12] G.-C. Liao, "Solve environmental economic dispatch of smart microgrid containing distributed generation system-Using chaotic quantum genetic algorithm," Electr. Power Energy Syst., vol. 43, no. 1, pp. 779-787, 2012.

[13] A. Takeuchi, T. Hayashi, Y. Nozaki, and T. Shimakage, "Optimal scheduling using metaheuristics for energy networks," IEEE Trans. Smart Grid, vol. 3, no. 2, pp. 968-974, Jun. 2012.

[14] R. Firestone and C. Marnay, "Energy manager design for microgrids," Lawrence Berkeley Nat. Lab., Berkeley, CA, USA, LBNL Rep. LBNL54447, 2005.

[15] A. Siddiqui, C. Marnay, O. Bailey, and K. LaCommare, "Optimal selection of on-site power generation with combined heat and power applications," Int. J. Distrib. Energy Resour., vol. 1, no. 1, pp. 33-62, 2005.

[16] G. Pepermans, J. Driesen, D. Haeseldonckx, R. Belmans, and W. D'haeseleer, "Distributed generation: Definition, benefits and issues," Energy Policy, vol. 33, no. 6, pp. 787-798, 2005.

[17] A. Siddiqui, C. Marnay, R. Firestone, and N. Zhou, "Distributed generation with heat recovery and storage," J. Energy Eng., vol. 133, no. 3, pp. 181-210, 2007.

[18] F. Mohamed, "Microgrid modelling and online management," Ph.D. dissertation, Faculty of Electronics, Communications and Automation, Helsinki Univ. Technol., Espoo, Finland, 2008.

[19] C. Chen, S. Duan, T. Cai, B. Liu, and G. Hu, "Smart energy management system for optimal micro grid economic operation," IET Renew. Power Generat., vol. 5, no. 3, pp. 258-267, 2011.

[20] P. Stluka, D. Godbole, and T. Samad, "Energy management for buildings and microgrids," in Proc. IEEE Conf. Decision Control, Orlando, FL, USA, Dec. 2011, pp. 5150-5157.

[21] B. Otomega, A. Marinakis, M. Glavic, and T. V. Cutsem, "Model predictive control to alleviate thermal overloads," IEEE Trans. Power Syst., vol. 22, no. 3, pp. 1384-1385, Aug. 2007.

[22] P. Meibom, R. Barth, B. Hasche, H. Brand, C. Weber, and M. O'Malley, "Stochastic optimization model to study the operational impacts of high wind penetrations in ireland," IEEE Trans. Power Syst., vol. 26, no. 3, pp. 1367-1379, Aug. 2011.

[23] M. Falahi, K. Butler-Purry, and M. Ehsani, "Dynamic reactive power control of islanded microgrids," IEEE Trans. Power Syst., vol. 28, no. 4, pp. 3649-3657, Nov. 2013. 
[24] G. Ferrari-Trecate, E. Gallestey, P. Letizia, M. Spedicato, M. Morari, and M. Antoine, "Modeling and control of co-generation power plants: A hybrid system approach," IEEE Trans. Control Syst. Technol., vol. 12, no. 5, pp. 694-705, Sep. 2004.

[25] L. Xie and M. Ilic, "Model predictive economic/environmental dispatch of power systems with intermittent resources," in Proc. IEEE Power Energy Soc. General Meeting, Jul. 2009, pp. 1-6.

[26] R. Negenborn, M. Houwing, J. D. Schutter, and J. Hellendoorn, "Model predictive control for residential energy resources using a mixed-logical dynamic model," in Proc. IEEE ICNSC, Okayama, Japan, Mar. 2009, pp. 702-707.

[27] P. Kriett and M. Salani, "Optimal control of a residential microgrid," Energy, vol. 42, no. 1, pp. 321-330, 2012

[28] A. Hooshmand, H. Malki, and J. Mohammadpour, "Power flow management of microgrid networks using model predictive control," Comput. Math. Appl., vol. 64, no. 5, pp. 869-876, 2012.

[29] X. Xia, J. Zhang, and A. Elaiw, "A model predictive control approach to dynamic economic dispatch problem," in Proc. IEEE Bucharest Power Tech Conf., Bucharest, Romania, Jun./Jul. 2009, pp. 1-7.

[30] W. Qi, J. Liu, X. Chen, and P. Christofides, "Supervisory predictive control of standalone wind/solar energy generation systems," IEEE Trans. Control Syst. Technol., vol. 19, no. 1, pp. 199-207, Jan. 2011.

[31] W. Qi, J. Liu, X. Chen, and P. Christofides, "Supervisory predictive control for long-term scheduling of an integrated wind/solar energy generation and water desalination system," IEEE Trans. Control Syst. Technol., vol. 20, no. 2, pp. 504-512, Mar. 2012.

[32] R. Palma-Behnke, C. Benavides, F. Lanas, B. Severino, L. Reyes, J. Llanos, et al., "A microgrid energy management system based on the rolling horizon strategy," IEEE Trans. Smart Grid, vol. 4, no. 2, pp. 996-1006, Jun. 2013.

[33] A. Bidram and A. Davoudi, "Hierarchical structure of microgrids control system," IEEE Trans. Smart Grid, vol. 3, no. 4, pp. 1963-1976, Dec. 2012.

[34] J. Maciejowski, Predictive Control with Constraints. Harlow, U.K.: Prentice-Hall, 2002.

[35] D. Mayne, "Constrained optimal control," in Proc. Eur. Control Conf., Plenary Lecture, Porto, Portugal, Sep. 2001.

[36] A. Bemporad and M. Morari, "Control of systems integrating logic, dynamics, and constraints," Automatica, vol. 35, no. 3, pp. 407-427, 1999.

[37] A. Parisio and L. Glielmo, "Energy efficient microgrid management using model predictive control," in Proc. 50th IEEE Conf. Decision Control, Orlando, FL, USA, Dec. 2011, pp. 5449-5454.

[38] M. Carriòn and J. Arroyo, "A computationally efficient mixed-integer linear formulation for the thermal unit commitment problem," IEEE Trans. Power Syst., vol. 21, no. 3, pp. 1371-1378, Aug. 2006.

[39] A. Richard and J. How, "Mixed-integer programming for control," in Proc. Amer. Control Conf., vol. 4. Portland, OR, USA, Jun. 2005, pp. 2676-2683.

[40] A. Bemporad, "Tutorial on model predictive control of hybrid systems," in Proc. Adv. Process Control Appl. Ind. Workshop, Vancouver, BC, Canada, 2007.

[41] D. Bertsimas and J. Tsitsiklis, Introduction to Linear Optimization. Belmont MA, USA: Athena Scientific, 1997.

[42] C. Floudas, Nonlinear and Mixed-Integer Programming-Fundamentals and Applications. Oxford, U.K.: Oxford Univ. Press, 1995.

[43] CPLEX 12.0 Users Manual, ILOG, Sunnyvale, CA, USA, 2012.

[44] "DC2: Evaluation of the microgrid central controller strategies. Microgrids-large scale integration of micro-generation to low voltage grids," Tech. Rep. ENK5-CT-2002-00610, 2004.

[45] Y. Yafeng, L. Yue, G. Junjun, and T. Chongli, "A new fuzzy neural networks model for demand forecasting," in Proc. IEEE Int. Conf. ICAL, Sep. 2008, pp. 372-376.

[46] C. Cortes, "Support-vector networks," Mach. Learn., vol. 20, no. 3, pp. 273-297, 1995

[47] E. Osuna, R. Freund, and F. Girosi, "Support vector machines: Training and applications," Comput. Sci. Artif. Intell. Lab (CSAIL), Massachusetts Institute of Technology, Cambridge, MA, USA, Tech. Rep. AIM-1602, Mar. 1997.
[48] L. Yue, Y. Yafeng, G. Junjun, and T. Chongli, "Demand forecasting by using support vector machine," in Proc. 3rd Int. Conf. Natural Comput., vol. 3. Aug. 2007, pp. 272-276.

[49] N. Sharma, P. Sharma, D. Irwin, and P. Shenoy, "Predicting solar generation from weather forecasts using machine learning," in Proc. 2nd IEEE Int. Conf. Smart Grid Commun., Brussels, Belgium, Oct. 2011, pp. 528-533.

[50] G. Nemhauser and L. Wolsey, Integer and Combinatorial Optimization. New York, NY, USA: Wiley, 1988.

[51] K. Pelckmans, J. Suykens, T. V. Gestel, J. D. Brabanter, L. Lukas, B. Hamers, et al., "LS-SVMlab: A MATLAB/C toolbox for least squares support vector machines," Tech. Rep., 1998.

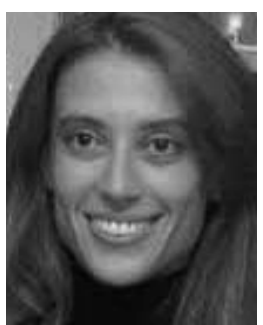

Alessandra Parisio (M'11) received the Ph.D degree in computer science engineering from the University of Sannio, Benevento, Italy.

She was with the Swiss Federal Institute of Technology, Zurich, Switzerland, where she was involved in the research project use of weather and occupancy forecasts for optimal building climate control. She is currently a Post-Doctoral Fellow with the Automatic Control Laboratory, Royal Institute of Technology, Stockholm, Sweden. Her current research interests include scheduling of energy and manufacturing systems and stochastic constrained control.

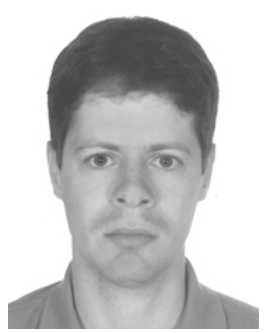

Evangelos Rikos received the Ph.D. degree in electrical and computer engineering from the University of Patras, Patras, Greece.

$\mathrm{He}$ is currently a Researcher with the Department of Photovoltaics and DER Systems, Centre for Renewable Energy Sources, Athens, Greece. He was a Lecturer with the University of Patras and he is a member of the Technical Chamber of Greece. His current research interests include renewable energy sources and especially photovoltaic systems, distributed generation, microgrids, energy efficiency, power electronics, and electromotion systems in electric vehicles.

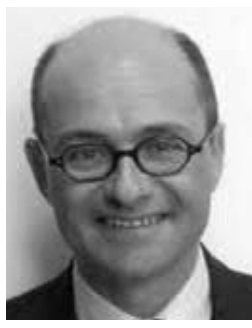

Luigi Glielmo (SM'83) received the Laurea degree in electronic engineering and the Research Doctorate degree in automatic control from the University of Naples Federico II

He taught at the University of Palermo, Palermo, Italy, University of Naples Federico II, Naples, Italy, and University of Sannio, Benevento, Italy, where he is currently a Professor of automatic control. He has co-authored more than 100 papers in international archival journals or proceedings of international conferences, co-edited two books, and holds three patents. His current research interests include singular perturbation methods, model predictive control methods, automotive controls, deep brain stimulation modeling and control, and smart-grid control. 